\title{
Validación de parrilla de contenidos y desarrollo de plataforma digital de libros ilustrados de no ficción *
}

\section{Content grid validation and digital platform development for non-fiction picture books}

\author{
Manuel Francisco Romero Oliva \\ Universidad de Cádiz \\ manuelfrancisco.romero@uca.es \\ ORCID ID: https://orcid.org/0000-0002-6854-0682
}

\author{
Hugo Heredia Ponce \\ Universidad de Cádiz \\ hugo.heredia@uca.es \\ ORCID ID: https://orcid.org/0000-0003-3657-1369
}

\section{Ester Trigo Ibáñez}

Universidad de Cádiz

ester.trigo@uca.es

ORCID ID: https://orcid.org/0000-0003-3035-4398

\section{Carmen Romero Claudio}

Universidad de Cádiz

carmen.romeroclaudio@gmail.com

ORCID ID: https://orcid.org/0000-0002-2813-9579

DOI: $10.17398 / 1988-8430.34 .143$

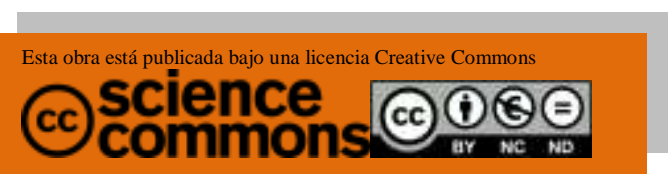

Fecha de recepción: $24 / 02 / 2021$ Fecha de aceptación: 31/03/2021

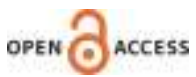

Romero Oliva, M. F.; Heredia Ponce, H.; Trigo Ibáñez, E., y Romero Claudio, C. (2021). Validación de parrilla de contenidos y desarrollo de plataforma digital de libros ilustrados de no ficción. Tejuelo, 34, 143-170.

Doi: https://doi.org/10.17398/1988-8430.34.143

\footnotetext{
" Este trabajo se encuadra dentro del proyecto I+D+I RTI2018-093825-B-I00 Formar lectores en la sociedad digital desde el libro de no ficción.
} 
Resumen: El desarrollo tecnológico de la sociedad actual ha provocado una modificación en los modos de lectura y, por consiguiente, en la concepción del libro. Esta circunstancia ha propiciado la expansión del mercado editorial. En este contexto, proliferan los libros ilustrados de no ficción, que plantean, desde una nueva concepción manipulativa de libro-objeto una hibridación entre lo analógico y lo digital para la divulgación del saber científico y el desarrollo paulatino de la competencia informacional de la infancia y la juventud. Si queremos que los libros ilustrados de no ficción cumplan su cometido, resulta oportuno analizar y sistematizar sus características. Por ello, en este trabajo se aporta el diseño y validación de una parrilla de análisis documental que permitirá analizar en profundidad los libros ilustrados de no ficción y se presenta la configuración de una plataforma digital interactiva que actuará como repositorio y portal virtual de encuentro para las personas interesadas en este tipo de libros. Para validar el instrumento se ha contado con el criterio de personas expertas en el ámbito que han aportado su visión crítica, lo que, junto al proceso de validación estadística, ha permitido configurar un instrumento fiable $y$ extrapolable a otros contextos de investigación.

Palabras clave: libro-objeto; lectura; literacidad; mediación lectora; repositorio digital.
Abstract: The technological development of today's society has caused a modification in the ways of reading and, consequently, in the conception of the book. This circumstance has led the expansion of the publishing market. In this context, non-fiction illustrated books proliferate, proposing, from a new manipulative conception of bookobject, a hybridization between analog and digital for the dissemination of scientific knowledge, and the gradual development of childhood and youth informational competence. If we want that illustrated non-fiction books fulfill their mission, it is appropriate to analyze and systematize their characteristics. For this reason, this work provides the design and validation of a content grid that will allow an in-depth analysis of nonfiction illustrated books and presents the configuration of an interactive digital platform that will act as a repository and meeting virtual portal for people interested in these types of books. To validate the instrument, we have relied on the criteria of experts in the field who have contributed their critical vision, which, together with the statistical validation process, has made possible to configure a reliable instrument that can be extrapolated to other research contexts.

Keywords: object-book; reading; literacy; reading mediation; digital repositor. 


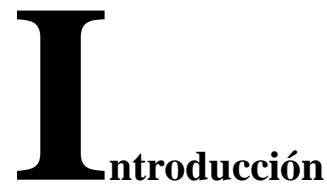

Leer en 2021 no puede limitarse a la primera acepción del Diccionario de la Lengua Española: "Pasar la vista por lo escrito o impreso, comprendiendo la significación de los caracteres empleados" (RAE, 2001), pues no cabe duda de que el desarrollo tecnológico de la sociedad actual ha provocado una modificación en los modos de lectura y, por consiguiente, en "la concepción del libro en todos sus niveles, desde el concepto de autoría, hasta su materialidad proponiendo una nueva experiencia de lectura" (Sampériz, Tabernero, Colón y Manrique, 2020, p. 74).

Este cambio de paradigma ha posibilitado, desde los albores del nuevo milenio, la expansión del mercado editorial. Es precisamente en este contexto en el que proliferan los libros ilustrados de no ficción, que plantean, desde una nueva concepción manipulativa de libro-objeto o libro-arte (Tabernero, 2018; 2019) una hibridación entre lo analógico y lo digital para la divulgación del saber científico, y, por ende, el 
desarrollo paulatino de la competencia informacional de la infancia y la juventud (Moss, 1991; Alexander y Jarman, 2018; Cordón-García, 2018).

Sin embargo, como señala Vigne (2017), "hay demasiados editores, pero también hay demasiados libros y, sobre todo, demasiados libros insignificantes y sin valor, sin originalidad, que se aniquilan entre sí" (p. 16). En este marco surge el proyecto "I+D+I RTI2018-093825B-I00 Formar lectores en la sociedad digital desde el libro de no ficción", donde se inserta este trabajo. Este proyecto, entre otros objetivos, se plantea estudiar el fenómeno editorial que rodea al libro ilustrado de no ficción y conceptualizarlo, situándolo respecto a la literatura infantil y juvenil; aportar bases teóricas para que los agentes mediadores puedan promover la lectura de este tipo de libros desde una visión analítica y crítica (Florido, 2020; Heredia y Sánchez-Herrero, 2020; Sampériz et al., 2020; Romero Oliva, Florido y Heredia, 2021a; Campos y Marcos, 2021; Romero Oliva, Trigo y Heredia, 2021b).

Si bien el libro ilustrado de no ficción constituye para algunos autores un subgénero de la literatura infantil y juvenil (Colman, 2007; Burgos, 2019), las claves para considerarlo un género independiente las aporta Lartitegui (2018, pp. 59-62) quien -aún reconociendo que no es descabellado incluir este tipo de libros en el ecosistema literario- parte de la idea de que ciencia y divulgación persiguen objetivos distintos pues la primera se centra en establecer leyes explicativas, constatar hipótesis, etc., mientras que la segunda pretende acercar los conocimientos científicos a los lectores y, para ello, utiliza diferentes formas de discurso donde el lenguaje literario cobra especial protagonismo. Es más, para esta autora el uso de recursos literarios en la divulgación es clave para su supervivencia y éxito entre el receptor infantil y juvenil, de ahí que los divulgadores consigan:

- Transmitir una información limitada, fiable y organizada (Garralón, 2013). 
- Acompañar y guiar hacia el conocimiento desde una voz cercana, más interesada en la persona que en los datos (Lartitegui, 2018).

- Presentar el conocimiento de una manera cuidada en todos sus elementos: dedicatoria, notas explicativas, títulos de las secciones, colofones... (Romero Oliva, Trigo, Heredia, Romero Claudio, 2021c).

- Activar la capacidad de evocación, pues utilizan un modo de expresión poética que juega con la forma de decir las cosas, las resonancias, el cruce de sentidos y la multiplicidad de interpretaciones (Kesler, 2012).

- Invitar a la curiosidad desde el juego y el descubrimiento mediante la integración de recursos lúdicos y artísticos (Sampériz et al., 2020).

En consonancia con lo expuesto anteriormente, partiendo de las características que Young, Moss y Cornwell (2007) atribuyen a este tipo de libros, consideramos, como constatan Romero Oliva et al. (2021b), que resultan de gran utilidad para la formación de lectores críticos desde una doble perspectiva: la intrínseca y la extrínseca.

Partiendo de la perspectiva intrínseca, directamente relacionada con las características del propio lector, estos libros ayudan a despertar la curiosidad desde la motivación de los lectores reacios atrayéndolos con soportes visuales y formatos atractivos además de combinar la lectura por placer con la lectura por información y la investigación (Brugarolas y Martin, 2002; Bortnem, 2008; Alexander y Jarman, 2018).

Por su parte, desde la perspectiva extrínseca, conectada con su entorno escolar y ámbito de aprendizaje, los libros ilustrados de no ficción contribuyen a desarrollar habilidades y estrategias de lectura crítica (Topping, 2015; Teaching, 2019), a la vez que amplían el conocimiento, de las áreas curriculares lingüísticas y no lingüísticas, presentado en los libros de texto -aportando vocabulario técnico y académico que no suele encontrarse en los textos narrativo-literarios- 
(Florido, 2020). Además, se erigen como un recurso para proporcionar una variedad de estructuras textuales y características que logran preparar a los niños y a las niñas para afrontar con éxito su futuro desarrollando su alfabetización informática e informacional (Hughes, 2006; Li, Beecher y Byeong-Young, 2018; Kuhn et al., 2017).

Como se ha expuesto, los libros ilustrados de no ficción cumplen una tarea primordial tanto en la formación de lectores como en el desarrollo de su espíritu científico desde la divulgación y adaptación al destinatario, manteniendo puertas abiertas, planteando curiosidades para seducir a la infancia y la juventud, invitándoles a discutir o a investigar más (Moss; 2003; Garralón, 2013).

De esta forma, el lector es el verdadero protagonista y se sitúa en el centro de toda mediación e interacción con el libro para su disfrute y acceso a la información (Romero Oliva et al., 2021b). Es precisamente en este escenario donde las relaciones hipertextuales e intertextuales cobran sentido pues, será necesario, por un lado, activar el conocimiento del mundo de niños y jóvenes, estableciendo puentes de unión con otras artes (Genette, 2000) y, por otro lado, poner en marcha estrategias de aproximación desde el uso de epitextos virtuales (Lluch, Tabernero y Calvo, 2015; Heredia y Sánchez-Herrero, 2020; Romero Oliva et al., 2021a).

Sin embargo, la mayoría de las investigaciones dedicadas a la formación de lectores se centran en la educación literaria (Romero Oliva y Trigo, 2019) y la mayor parte de las obras que pueblan las bibliotecas escolares son de carácter literario (Santos-Díaz, 2017). Además, entre las lecturas realizadas por los docentes activos y en formación no se registran libros de no ficción (Granado, 2014; Juárez, 2019; Castillo-Fadić y Sologuren-Insúa, 2020).

Por otro lado, si bien es cierto que ya desde los años 90 del pasado siglo en el panorama internacional se vienen analizando las características y posibilidades para utilizar los libros ilustrados de no ficción en las aulas (Moss, 1991, 2003; Ruth, 2009; White, 2011, entre 
otros), el panorama de investigación nacional aún adolece de una falta de estudios centrados en estos aspectos pues, aunque existen propuestas muy acertadas (Garralón, 2013; Lartitegui, 2018), todavía es necesario seguir indagando, conceptualizando y recogiendo las voces de todos los agentes implicados: el terreno editorial, las bibliotecas, las universidades, los centros escolares y las familias.

A este respecto, si queremos que los libros ilustrados de no ficción cumplan el cometido de formar a lectores competentes y preparados para adentrarse en la sociedad de la información, consideramos oportuno analizar y sistematizar sus características. Por ello, el objetivo principal del presente estudio se centra en el diseño y validación de una parrilla de análisis documental que permita analizar en profundidad los libros ilustrados de no ficción. Este objetivo principal se materializa en los siguientes objetivos específicos:

1. Analizar los aspectos de contenido (texto e ilustración) y su relación con los destinatarios lectores.

2. Analizar los aspectos de contenido (texto e ilustración) y su dinamización en la escuela.

3. Definir los parámetros fundamentales para la clasificación y evaluación de libros en una base de datos con la que interaccionar con los diferentes tipos de usuarios de una forma efectiva.

De esta forma, el instrumento diseñado, que consideramos de gran utilidad para las personas interesadas en los libros ilustrados de no ficción, está dividido en 4 dimensiones:

- Dimensión 1: Ficha técnica del libro, donde se indican los aspectos referidos a datos propios del libro respecto a la autoría y la editorial.

- Dimensión 2: El libro objeto, donde se presta atención a los aspectos físicos externos e internos de la publicación.

- Dimensión 3: Contenido del libro, donde se analizan las características del texto, la tipología, las ilustraciones y sus posibles destinatarios. 
- Dimensión 4: Libro y escuela, donde se ofrece una perspectiva para su inclusión en los procesos educativos y curriculares de las distintas etapas educativas.

\section{Metodología}

\subsection{Participantes}

En este proceso de validación participaron seis jueces expertos de las universidades de Zaragoza y Cádiz. Todos de ellos son, bien integrantes del grupo de investigación (INEDLL) Investigación en Didáctica de la Lengua y la Literatura (Universidad de Cádiz) o bien del grupo (ECOLIJ) Educación Comunicativa y Literaria en la Sociedad de la Información. Literatura Infantil y Juvenil en la construcción de identidades (Universidad de Zaragoza), con una amplia formación en lo que se refiere a libros ilustrados de no ficción, principalmente motivada por su participación en el proyecto " $\mathrm{I}+\mathrm{D}+\mathrm{I}$ RTI2018-093825-B-I00 Formar lectores en la sociedad digital desde el libro de no ficción" y por su acercamiento a este tipo de libros desde la formación inicial de docentes a través de la realización de sus Trabajos Fin de Grado bajo la dirección de investigadores integrantes del proyecto. En la Tabla 1 se exponen sus perfiles:

\section{Tabla 1}

Información de los jueces expertos

\begin{tabular}{ccccc}
\hline Jueces & Sexo & Universidad & Formación & $\begin{array}{c}\text { Pertenencia al } \\
\text { proyecto }\end{array}$ \\
\hline 1 & Mujer & Cádiz & $\begin{array}{c}\text { Grado en Educación } \\
\text { Infantil }\end{array}$ & No \\
2 & Hombre & Cádiz & $\begin{array}{c}\text { Grado en Educación } \\
\text { Infantil }\end{array}$ & No \\
3 & Mujer & Zaragoza & Doctora & Sí \\
4 & Mujer & Zaragoza & Doctora & Sí \\
5 & Mujer & Zaragoza & Doctora & Sí \\
6 & Mujer & Zaragoza & Doctoranda & Sí \\
\hline
\end{tabular}

Fuente: Elaboración propia 
Para analizar el conocimiento de los jueces con respecto al contenido, se calculó su coeficiente de competencia $(\mathrm{K})$, ya utilizado en investigaciones precedentes como la realizada por Cabero y Barroso (2013). Este valor se obtiene a partir del cálculo, por una parte, del coeficiente de conocimiento $(\mathrm{Kc})$ a través de una escala del 1-10 y, por otra parte, del análisis de su coeficiente de argumentación (Ka) -vid. Tabla 2- a través de una escala del 1-3 con valores según las fuentes de argumentación que detallamos. Tras los resultados, se puede calcular $\mathrm{K}=0.5[\mathrm{kc}(0.10)+\mathrm{Ka}]$. De esta manera, se considera que un juez tiene competencia suficiente cuando el valor $\mathrm{K}$ se sitúa en el nivel alto, como se determina en la tabla 2 respecto a los criterios de dominio:

\section{Tabla 2}

\section{Coeficiente de argumentación}

Fuentes de argumentación

Grado de influencia de cada una de las fuentes en sus criterios

\begin{tabular}{lccc}
\hline \multicolumn{1}{c}{ Alto } & Medio & Bajo \\
\hline $\begin{array}{l}\text { Análisis teórico sobre los libros de } \\
\text { no ficción }\end{array}$ & 0.3 & 0.2 & 0.1 \\
$\begin{array}{l}\text { Su experiencia en cuanto a los } \\
\text { libros de no ficción en su práctica } \\
\text { docente }\end{array}$ & 0.5 & 0.4 & 0.2 \\
$\begin{array}{l}\text { Conocimiento de la literatura } \\
\text { nacional sobre los libros de ficción }\end{array}$ & 0.05 & 0.05 & 0.05 \\
$\begin{array}{l}\text { Conocimiento de la literatura } \\
\text { internacional sobre los libros de no } \\
\text { ficción } \\
\begin{array}{l}\text { Conocimiento propio sobre } \\
\text { fomento de la lectura }\end{array}\end{array}$ & 0.05 & 0.05 & 0.05 \\
\begin{tabular}{l} 
Intuición del experto \\
\hline
\end{tabular}
\end{tabular}

Fuente: Elaboración propia 


\subsection{Instrumento}

Se ha escogido una parrilla de análisis porque este instrumento permite organizar, de manera sistematizada y en diferentes categorías, los elementos -en forma de ítems- que, divisados desde una visión de conjunto, configuran el universo objeto de estudio: los libros ilustrados de no ficción (López Noguero, 2002). Así, en esta parrilla se incorporan ítems cualitativos y cuantitativos -vid. Tabla 3- distribuidos en 4 dimensiones bien diferenciadas, pero entrelazadas entre sí.

Tabla 3

Distribución parrilla

\begin{tabular}{ccc}
\hline Tipos & $\mathbf{N}^{\mathbf{0}}$ & Ítems \\
\hline Cerrados & 98 & $1-6 ; 15$ \\
Abiertos & 7 & $7-14 ; 16-105$ \\
\hline
\end{tabular}

Fuente: Elaboración propia

\subsection{Procedimiento}

La validación y elaboración de este cuestionario se desarrolló siguiendo los pasos que establecen Agreda, Hinojo y Sola (2016). En primer lugar, se establecieron las dimensiones que habría de contener la parrilla; seguidamente, se redactaron los ítems integrados en cada una de ellas; posteriormente, se procedió a realizar una validación de contenido contando con el juicio de un grupo de expertos cuya formación disciplinar quedó constatada; a continuación, una vez analizadas las aportaciones de los expertos, se realizó una serie de modificaciones que culminarían en la construcción de una parrilla validada; y, finalmente, se transfirió el instrumento generado a un repositorio digital. 


\section{Figura 1}

Procedimiento de la validación

105 ítems (ver anexo)

D1. 21 ítems

D2. 18 ítems

D3. 47 ítems

D4. 19 items
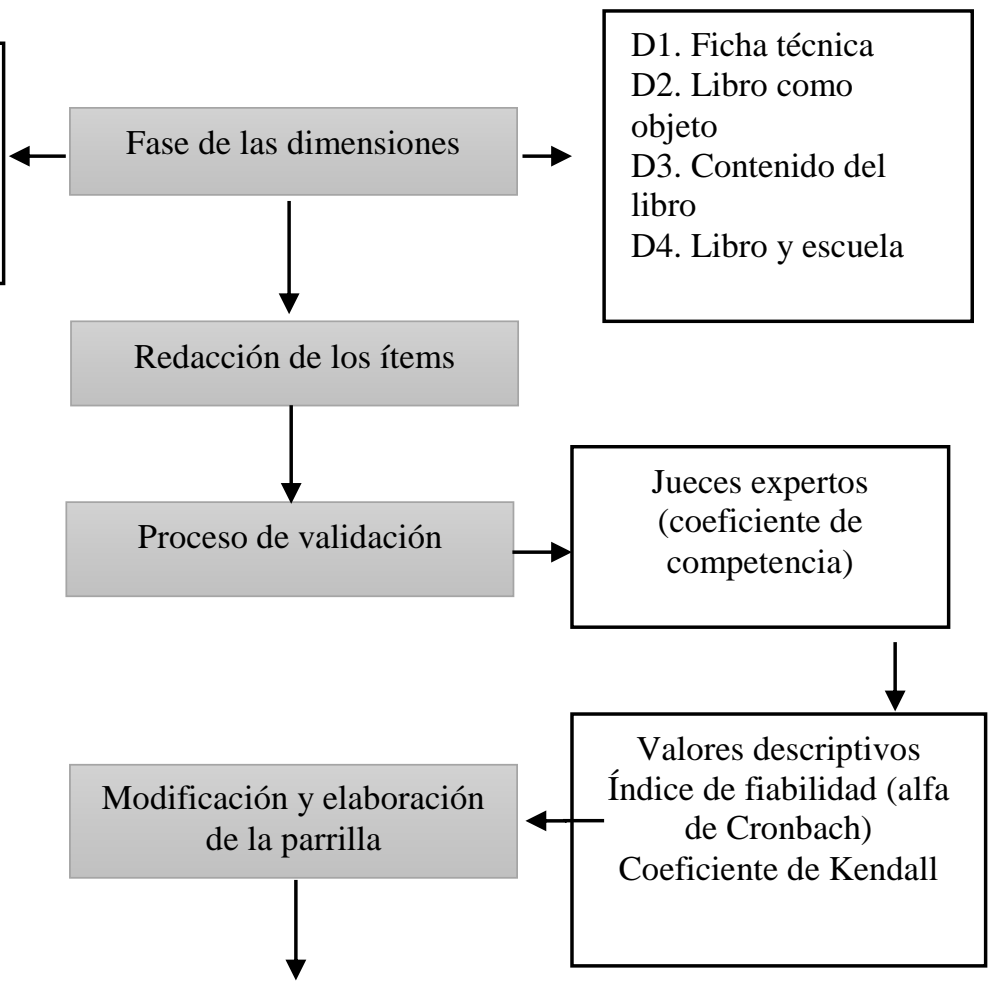

Repositorio digital

Administración de la

parrilla

Fuente: Elaboración propia. Adaptación de Agreda et al. (2016)

En primera instancia, se ofreció a los jueces una planilla en la que debían valorar los distintos ítems teniendo en cuenta su claridad y pertinencia dentro de la dimensión en la que se consignaban utilizando, siguiendo el criterio de Matas (2018), una escala Likert de 5 valores. De 
esta forma, "esta escala incluía un punto medio neutral, así como puntos a izquierda y derecha, originalmente de desacuerdo y de acuerdo, con opciones de respuesta numéricas de 1 a 5 " (p. 2). Además, se ofrecía un espacio para que los jueces pudieran realizar observaciones y propuestas de cambio. La información obtenida se organizó en una matriz diseñada ad hoc. Para analizar los diferentes resultados, se utilizó el paquete estadístico Statistical Package for the Social Sciences (SPSS), versión 24.

\section{Resultados}

A continuación, comentaremos los diferentes resultados siguiendo las fases expuestas anteriormente -vid. Figura 1-, centrándonos en el proceso de validación.

Antes de poder validar la parrilla, tuvimos que escoger a los diferentes jueces expertos para este cometido. Para ello, se calculó el valor K. Así, los resultados correspondientes a cada juez - vid. Tabla 4fueron los siguientes:

\section{Tabla 4}

Coeficiente de competencia

\begin{tabular}{ccccc}
\hline Jueces & Kc & Ka & K & Conclusión \\
\hline 1 & 0.7 & 0.6 & $\mathbf{0 . 6 5}$ & Medio \\
2 & 0.7 & 0.6 & $\mathbf{0 . 6 5}$ & Medio \\
3 & 1 & 1 & $\mathbf{1}$ & Alto \\
4 & 0.9 & 1 & $\mathbf{0 . 9 5}$ & Alto \\
5 & 0.9 & 0.8 & $\mathbf{0 . 8 5}$ & Alto \\
6 & 0.8 & 0.9 & $\mathbf{0 . 8 5}$ & Alto \\
\hline
\end{tabular}

Fuente: Elaboración propia

Según estos resultados, podemos decir que el valor $\mathrm{K}$ sobre el tema que tiene cada juez es adecuado y, por tanto, se consideran válidos para afrontar esta tarea. Sin embargo, aunque, como puede apreciarse, los dos primeros informantes ofrecen valores por debajo de 0.8 (en concreto, 0.65) se seleccionaron porque nuestra intención era obtener la visión de los estudiantes en formación. El resto de los informantes $(3,4$, 
5 y 6 ) en cambio, ofrecen valores por encima de 0.8 , lo que constata su idoneidad para ejercer como sujetos validadores.

En cuanto a los resultados de valoración de la parrilla, se obtuvieron los siguientes:

En lo concerniente a las dimensiones, observamos que todas alcanzan puntuaciones medias superiores a 4 -vid. Tabla 5-, por lo tanto, se podrían considerar aceptables:

\section{Tabla 5}

Datos descriptivos de las dimensiones

\begin{tabular}{cccccc}
\hline Dimensiones & N & Mínimo & Máximo & Media & $\begin{array}{c}\text { Desviación } \\
\text { estándar }\end{array}$ \\
\hline 1 & 6 & 4.33 & 5.00 & 4.7143 & .28887 \\
2 & 6 & 4.44 & 5.00 & 4.8333 & .20488 \\
3 & 6 & 4.30 & 5.00 & 4.7092 & .26767 \\
4 & 6 & 4.26 & 4.95 & 4.6842 & .26210 \\
$\begin{array}{c}\text { N válido (por } \\
\text { lista) }\end{array}$ & 6 & & & & \\
\hline
\end{tabular}

Fuente: Elaboración propia. Extraídos de SPSS

Una vez que observamos que las dimensiones estadísticamente aportaban una media óptima, procedimos a calcular el índice de fiabilidad de estas a través del coeficiente alfa de Cronbach -vid. Tabla 6-.

\section{Tabla 6}

Alfa de Cronbach

Fuente: Elaboración propia. Extraídos de SPSS

\begin{tabular}{cr}
$\begin{array}{c}\text { Alfa de } \\
\text { Cronbach }\end{array}$ & \multicolumn{2}{c}{$\begin{array}{c}\text { N de } \\
\text { elementos }\end{array}$} \\
\hline .928 & 4 \\
\hline
\end{tabular}

En este sentido, sabiendo que el coeficiente de alfa tiene un valor de ,928 podemos indicar, siguiendo a George y Mallery (2003), 
que es excelente porque .928>.9 a .95. Si vemos este dato a través de cada dimensión, apreciamos que, si eliminásemos la dimensión 2, -vid. Tabla 7-, el alfa de Cronbach subiría, pero, al no ser muy significativa esa subida y encontrarse dentro del intervalo "excelente", decidimos no eliminarla:

\section{Tabla 7}

Alfa de Cronbach si se elimina una dimensión

\begin{tabular}{ccccc}
\hline Dimensiones & $\begin{array}{c}\text { Media de } \\
\text { escala si el } \\
\text { elemento se } \\
\text { ha }\end{array}$ & $\begin{array}{c}\text { Varianza de } \\
\text { escala si el } \\
\text { elemento se } \\
\text { haprimido }\end{array}$ & $\begin{array}{c}\text { Correlación } \\
\text { total de } \\
\text { elementos } \\
\text { corregida }\end{array}$ & $\begin{array}{c}\text { Alfa de } \\
\text { Cronbach si } \\
\text { el elemento } \\
\text { se ha } \\
\text { suprimido }\end{array}$ \\
\hline 1 & 14.2268 & .445 & .895 & .886 \\
2 & 14.1077 & .596 & .744 & .937 \\
3 & 14.2318 & .497 & .808 & .914 \\
4 & 14.2568 & .475 & .916 & .877 \\
\hline
\end{tabular}

Fuente: Elaboración propia. Extraídos de SPSS

Además, nos interesaba conocer el índice de fiabilidad por cada ítem y observamos que el alfa de Cronbach era de .962 "excelente", y que, si eliminásemos alguno, el cambio no sería significativo -vid. Tabla 8 . 
Tabla 8

Alfa de Cronbach si se elimina un ítem

\begin{tabular}{|c|c|c|c|c|}
\hline Ítems & $\begin{array}{c}\text { Media de } \\
\text { escala si el } \\
\text { elemento se } \\
\text { ha suprimido }\end{array}$ & $\begin{array}{c}\text { Varianza de } \\
\text { escala si el } \\
\text { elemento se } \\
\text { ha suprimido }\end{array}$ & $\begin{array}{c}\text { Correlación } \\
\text { total de } \\
\text { elementos } \\
\text { corregida }\end{array}$ & $\begin{array}{c}\text { Alfa de } \\
\text { Cronbach si } \\
\text { el elemento } \\
\text { se ha } \\
\text { suprimido } \\
\end{array}$ \\
\hline P1 & 491.5000 & 633.100 & -.185 & .963 \\
\hline $\mathrm{P} 2$ & 491.6667 & 625.867 & .129 & .963 \\
\hline P3 & 491.8333 & 619.767 & .216 & .963 \\
\hline $\mathrm{P} 4$ & 491.5000 & 629.900 & -.029 & .963 \\
\hline P5 & 491.6667 & 605.867 & .918 & .961 \\
\hline P6 & 491.6667 & 617.067 & .473 & .962 \\
\hline P7 & 491.8333 & 606.167 & .853 & .961 \\
\hline P8 & 491.6667 & 617.067 & .473 & .962 \\
\hline P9 & 491.8333 & 606.167 & .853 & .961 \\
\hline P10 & 491.5000 & 618.700 & .522 & .962 \\
\hline P11 & 491.5000 & 618.700 & .522 & .962 \\
\hline P12 & 491.5000 & 618.700 & .522 & .962 \\
\hline P13 & 491.5000 & 618.700 & .522 & .962 \\
\hline P14 & 491.5000 & 618.700 & .522 & .962 \\
\hline P15 & 491.8333 & 593.367 & .868 & .961 \\
\hline P16 & 491.6667 & 617.067 & .473 & .962 \\
\hline P17 & 491.6667 & 605.867 & .918 & .961 \\
\hline P18 & 491.5000 & 616.700 & .621 & .962 \\
\hline P19 & 491.5000 & 616.700 & .621 & .962 \\
\hline P20 & 491.6667 & 617.067 & .473 & .962 \\
\hline $\mathrm{P} 21$ & 491.5000 & 616.700 & .621 & .962 \\
\hline $\mathrm{P} 22$ & 491.5000 & 616.700 & .621 & .962 \\
\hline P23 & 491.5000 & 629.900 & -.029 & .963 \\
\hline $\mathrm{P} 24$ & 491.5000 & 633.100 & -.185 & .963 \\
\hline $\mathrm{P} 25$ & 491.5000 & 633.100 & -.185 & .963 \\
\hline P26 & 491.5000 & 616.700 & .621 & .962 \\
\hline $\mathrm{P} 27$ & 491.5000 & 616.700 & .621 & .962 \\
\hline P28 & 491.5000 & 633.100 & -.185 & .963 \\
\hline P29 & 491.5000 & 616.700 & .621 & .962 \\
\hline P30 & 491.5000 & 618.700 & .522 & .962 \\
\hline P31 & 491.5000 & 629.900 & -.029 & .963 \\
\hline P32 & 491.5000 & 629.900 & -.029 & .963 \\
\hline P33 & 491.5000 & 618.700 & .522 & .962 \\
\hline P34 & 491.5000 & 616.700 & .621 & .962 \\
\hline P35 & 491.5000 & 616.700 & .621 & ,962 \\
\hline
\end{tabular}




\begin{tabular}{|c|c|c|c|c|}
\hline P36 & 491.5000 & 616.700 & .621 & .962 \\
\hline P37 & 491.5000 & 616.700 & .621 & .962 \\
\hline P38 & 491.5000 & 616.700 & .621 & .962 \\
\hline P39 & 491.5000 & 616.700 & .621 & .962 \\
\hline P40 & 491.8333 & 634.167 & -.128 & .964 \\
\hline P41 & 491.6667 & 605.867 & .918 & .961 \\
\hline P42 & 491.5000 & 618.700 & .522 & .962 \\
\hline P43 & 491.5000 & 618.700 & .522 & .962 \\
\hline P44 & 491.5000 & 618.700 & .522 & .962 \\
\hline P45 & 491.8333 & 619.767 & .216 & .963 \\
\hline P46 & 491.6667 & 619.067 & .394 & .962 \\
\hline P47 & 491.5000 & 618.700 & .522 & .962 \\
\hline P48 & 491.5000 & 618.700 & .522 & .962 \\
\hline P49 & 491.5000 & 618.700 & .522 & .962 \\
\hline P50 & 491.5000 & 618.700 & .522 & .962 \\
\hline P51 & 491.5000 & 616.700 & .621 & .962 \\
\hline P52 & 491.6667 & 633.467 & -.164 & .963 \\
\hline P53 & 491.8333 & 604.567 & .588 & .962 \\
\hline P54 & 491.8333 & 611.767 & .411 & .962 \\
\hline P55 & 491.6667 & 619.067 & .394 & .962 \\
\hline P56 & 491.5000 & 618.700 & .522 & .962 \\
\hline P57 & 491.8333 & 611.767 & .411 & .962 \\
\hline P58 & 491.6667 & 617.067 & .473 & .962 \\
\hline P59 & 491.8333 & 607.767 & .509 & .962 \\
\hline P60 & 491.6667 & 619.067 & .394 & .962 \\
\hline P61 & 491.6667 & 617.067 & .473 & .962 \\
\hline P62 & 491.6667 & 642.267 & .499 & .964 \\
\hline P63 & 491.5000 & 616.700 & .621 & .962 \\
\hline P64 & 491.5000 & 616.700 & .621 & .962 \\
\hline P65 & 491.5000 & 616.700 & .621 & .962 \\
\hline P66 & 491.5000 & 616.700 & .621 & .962 \\
\hline P67 & 491.5000 & 616.700 & .621 & .962 \\
\hline P68 & 491.6667 & 605.867 & .918 & .961 \\
\hline P69 & 491.8333 & 634.167 & -.128 & .964 \\
\hline P70 & 491.6667 & 620.267 & .347 & ,962 \\
\hline P71 & 491.6667 & 605.867 & .918 & .961 \\
\hline P72 & 491.5000 & 618.700 & .522 & .962 \\
\hline P73 & 491.5000 & 618.700 & .522 & .962 \\
\hline P74 & 491.5000 & 618.700 & .522 & .962 \\
\hline P75 & 491.5000 & 618.700 & .522 & .962 \\
\hline P76 & 491.6667 & 620.267 & .347 & .962 \\
\hline P77 & 491.8333 & 611.767 & .411 & .962 \\
\hline P78 & 491.6667 & 622.267 & .269 & .962 \\
\hline P79 & 491.5000 & 618.700 & .522 & .962 \\
\hline
\end{tabular}




\begin{tabular}{lllll} 
P80 & 491.6667 & 605.867 & .918 & .961 \\
P81 & 491.8333 & 593.367 & .868 & .961 \\
P82 & 491.6667 & 605.867 & .918 & .961 \\
P83 & 491.6667 & 605.867 & .918 & .961 \\
P84 & 491.5000 & 616.700 & .621 & .962 \\
P85 & 491.6667 & 627.867 & .052 & .963 \\
P86 & 491.5000 & 618.700 & .522 & .962 \\
P87 & 491.6667 & 605.867 & .918 & .961 \\
P88 & 491.6667 & 605.867 & .918 & .961 \\
P89 & 491.5000 & 618.700 & .522 & .962 \\
P90 & 491.6667 & 604.267 & .611 & .962 \\
P91 & 491.5000 & 618.700 & .522 & .962 \\
P92 & 491.8333 & 593.367 & .868 & .961 \\
P93 & 491.6667 & 605.867 & .918 & .961 \\
P94 & 491.8333 & 624.167 & .110 & .963 \\
P95 & 491.6667 & 605.867 & .918 & .961 \\
P96 & 491.8333 & 651.767 & -.538 & .965 \\
P97 & 491.6667 & 617.067 & .473 & .962 \\
P98 & 491.6667 & 622.267 & .269 & .962 \\
P99 & 491.5000 & 638.700 & -.456 & .963 \\
P100 & 491.5000 & 616.700 & .621 & .962 \\
P101 & 491.5000 & 616.700 & .621 & .962 \\
P102 & 491.8333 & 634.167 & -.128 & .964 \\
P103 & 491.5000 & 616.700 & .621 & .962 \\
P104 & 491.6667 & 605.867 & .918 & .961 \\
P105 & 491.6667 & 629.867 & -.026 & .963 \\
\hline
\end{tabular}

Fuente: Elaboración propia. Extraídos de SPSS

Tras haber constatado que el índice de fiabilidad es aceptable, quisimos analizar el nivel de concordancia que tienen los jueces expertos a través del coeficiente de Kendall -vid. Tabla 9-.

\section{Tabla 9}

Coeficiente de Kendall

\begin{tabular}{ccccc}
\hline $\mathbf{N}$ & W de Kendall & Chi-cuadrado & gl & Sig. asintótica \\
\hline 6 & .553 & 6.632 & 2 & .36 \\
\hline
\end{tabular}

Fuente: Elaboración propia. Extraídos de SPSS

De este modo, la Tabla 9 muestra que existe una alta concordancia entre las valoraciones que los jueces han ofrecido porque p>0.05 (0.36>0.05). En este sentido, siguiendo a López de Ullibarri y 159 | Pági n a 
Pita Fernández (1999), ese nivel de concordancia es mayor pues el valor W de Kendall está entre ,41 y ,60 $(, 41<, 55<, 60)$

Por lo tanto, vemos que la parrilla ha quedado validada y resulta muy adecuada para el uso por parte de la comunidad investigadora. En cambio, desde una visión cualitativa, los jueces expertos nos realizaron algunas aportaciones de mejora que nos resultarían muy útiles de cara a generar la versión digital de la parrilla y construir la plataforma digital.

Así, en la Figura 2 se aporta un diagrama de flujos en el que se muestra cómo las aportaciones realizadas por los jueces validadores hicieron que se vieran modificadas las dimensiones inicialmente proyectadas.

De este modo, algunos ítems se vieron, bien eliminados, bien desplazados de una dimensión a otra al considerarse más pertinentes dentro de la nueva ubicación o bien reformulados, según se recoge en la Tabla 10.

\section{Tabla 10}

Cambios de títulos de los ítems

\begin{tabular}{ccll}
\hline Dimensión & Ítems & \multicolumn{1}{c}{ Antes } & \multicolumn{1}{c}{ Ahora } \\
\hline 2 & P9 & Tipo de libro: otros & Tipo de libro: libro mudo \\
3 & P47 & $\begin{array}{l}\text { Destinatarios prelectores } \\
\text { Nivel verbal prelectores }\end{array}$ \\
3 & P48 & $\begin{array}{l}\text { Destinatarios lectores en } \\
\text { formación } \\
\text { Destinatarios } \\
\text { autónomos lectores } \\
\text { Nivel léxico gramatical }\end{array}$ & $\begin{array}{l}\text { Nivel verbal lectores en } \\
\text { formación } \\
\text { autónomos } \\
\text { Nivel léxico científico }\end{array}$ \\
3 & P49 léfico \\
3 & P51 & $\begin{array}{l}\text { Nivel léxico gramatical } \\
\text { literario }\end{array}$ & Nivel léxico literario \\
3 & P25 & $\begin{array}{l}\text { Nivel léxico gramatical } \\
\text { común Nivel léxico común } \\
\text { Tipología literaria }\end{array}$ & Tipología lírica \\
\hline
\end{tabular}

Fuente: Elaboración propia 


\section{Figura 2}

Distribuciones de los ítems por dimensiones

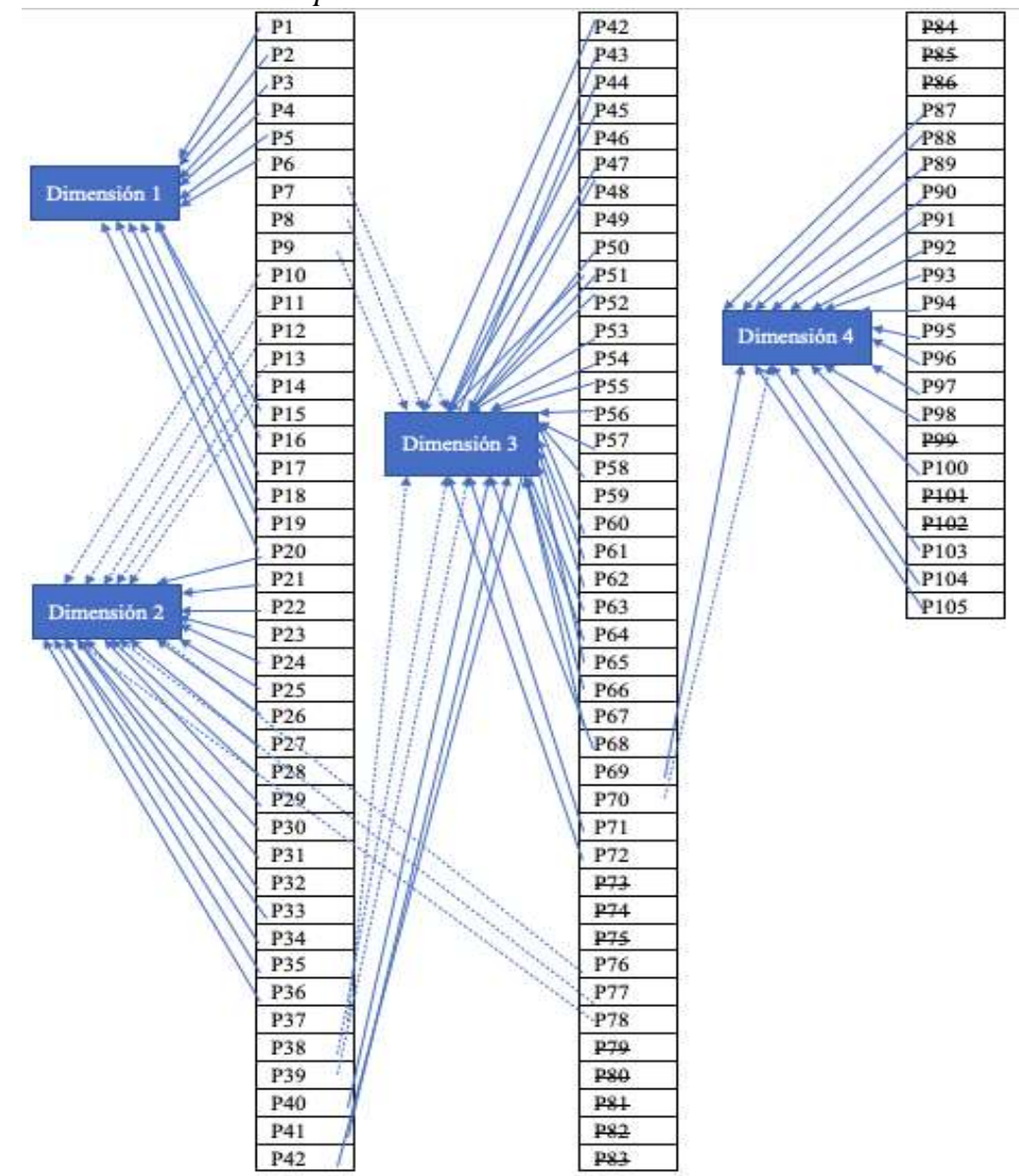

$\longrightarrow$ Ese ítem se mantiene en la dimensión inicial

$\rightarrow \quad$ Ese ítem cambia de dimensión

$3 \quad$ Se elimina ese ítem

Fuente: Elaboración propia

Además de lo anterior, se incluyeron nuevos ítems en las dimensiones 3 y 4 para hacer un análisis más exhaustivo: 
- Género no ficción pura

\section{Dimensión 3}

- Género mezcla ficción y no ficción

- Tipo de libro: libro ilustrado

- Tipo de libro: libro álbum

- Tipo de libro: libro mudo

- Información: informativo-expositivo

- Información: narrativo

- Información: cómic

- Información: otros

- La voz del texto: se da información sobre los autores

- La voz del texto: se presenta desde una voz científica

- Se presenta desde una voz divulgativa

- La voz del texto: se presenta en proceso de descubrimiento

- La voz del texto: otros

- Enfoque de la información: se sugiere completar la información con otras fuentes de información

- Enfoque de la información: se sugiere realizar determinadas actividades, experimentos complementarios

\section{Dimensión 4}

- Tipo de lectura que el libro propone: fragmentada

- Tipo de lectura que el libro propone: personal

- Tipo de lectura que el libro propone: seguida

Por lo tanto, tras la validación de la parrilla, como prospección y administración de esta, se desarrolló un repositorio digital donde cualquier usuario pudiese incorporar un libro de no ficción tomando como referencia todas las características que hemos establecido desde los ítems y que se ven reflejados en la plataforma -vid. Figura 3-.

De esta forma, los usuarios interesados en este tipo de libros pueden consultar, realizar búsquedas filtradas, proponer nuevos títulos, contactar con los integrantes del proyecto, aclarar dudas... Se trata de acercar lo máximo posible la investigación a la sociedad, realizando una transferencia directa y en consonancia con los hábitos de consumo informacional que van pautando los modelos de vida vigentes. 
Se trata, pues, de una plataforma de fácil manejo y acceso, con una apariencia muy similar a las de televisión o a las de ventas de productos multimarca, en las que aparecen recomendaciones basadas en los productos consultados o consumidos por los usuarios, valoraciones de otras personas, etc.

\section{Figura 3}

Distribución de los ítems en la plataforma

\begin{tabular}{|c|c|c|c|c|c|c|c|c|c|}
\hline \multirow{3}{*}{$l_{-1}$} & & foto & \multicolumn{2}{|l|}{ blob } & 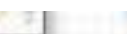 & \multirow{3}{*}{\multicolumn{2}{|c|}{ Bopectom Matoriales }} & \multirow{2}{*}{8} & \\
\hline & & id genero & \multicolumn{2}{|l|}{ integor } & $\geqslant$ & & & & \\
\hline & & lid_aspe mate & integer & - & 3 & & id & integer & $=2$ \\
\hline & & id_Justracion & integer & + & 7 & & formato & string. & $2=$ \\
\hline & & id tercto & integer & - & $\geqslant$ & & tamano & string & $=8$ \\
\hline & & id parateato & integer & 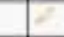 & 3 & & papel & string & $=$ \\
\hline & & id_lectura & integer & 2 & 7 & & paginas & integer & $2=$ \\
\hline & & id_cant curri & intoger & - & $\geqslant$ & & analogeco & text & -8 \\
\hline & \multirow{2}{*}{\multicolumn{2}{|c|}{ sodsdfield }} & & & & & Sigital & teot & $=$ \\
\hline \multirow{8}{*}{ 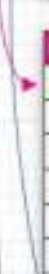 } & & & & & & & lome & binary & +2 \\
\hline & \multicolumn{4}{|c|}{ Huntracion } & & & portada & binary & -4 \\
\hline & 9 & \multicolumn{2}{|l|}{14} & integer & $Y \div$ & & contraportada & Binary & $=$ \\
\hline & & \multicolumn{2}{|l|}{ redundancis } & thinary & 23 & & cubierta & binary & $2=$ \\
\hline & & \multicolumn{2}{|c|}{ complementariedad } & binary & $2=$ & & id_cbese_es & integer & -3 \\
\hline & & \multicolumn{2}{|l|}{ dispuncion } & binary & $z=1$ & & guardas & Binary & $=$ \\
\hline & & \multicolumn{2}{|l|}{ id_obse } & teger. & 27 & & reapeto & binary & -2 \\
\hline & & id aspecton & & tegor - & 2 & & portadila & binary & -2 \\
\hline & sis & Adsifield & & & & & Irontis : & binary & $=$ \\
\hline & & & & & & & portada & binary & -2 \\
\hline & Teat & & & E & 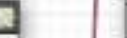 & & derechos & binary & -8 \\
\hline 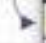 & 2 & id & integser & -2 & & & dedicsteria & binary & $=$ \\
\hline & & infermacion & string & 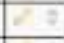 & & & peologg & binary & -2 \\
\hline & & facilitadores & string & 22 & & & peetiminares & binary & -8 \\
\hline & & niv_vert & string & $2=$ & & & indice & binary & $=$ \\
\hline & & glos_prin & binaty & $2=$ & & & coloton & binary & $=2$ \\
\hline & & glos, in & binary & 22 & & & id_abse in & integer & -4 \\
\hline & & gios fin & binary & 23 & & & id_aspectos: & integer & $=$ \\
\hline & & niv_lex & string & 23 & & & Add field & & \\
\hline & & estructura & string & 20 & & & & & \\
\hline & & tipolopia & string & $2=$ & & Para & atexto & & \\
\hline & & vat & string & $2=$ & & 2 & id & integer & 23 \\
\hline & & enfoque & string & 2 & & & booktrailer & string & $-=$ \\
\hline & & id_arsectos & integser & $-2=$ & & & Hiog & string & 2 \\
\hline & 34 & sdidfield & & & & & critica & string & $=2$ \\
\hline & & & & & & -36 & Add field & & \\
\hline
\end{tabular}

\begin{tabular}{|c|c|c|c|}
\hline \multicolumn{4}{|c|}{ Valoracien } \\
\hline 3 & id & integer & -3 \\
\hline & id_libro & integet & -4 \\
\hline & valor & integer & -2 \\
\hline & comentano & text & 27 \\
\hline & Astigid & & \\
\hline
\end{tabular}

\section{Cantenicasciniculare:}

$\rightarrow$ is

\begin{tabular}{|c|c|c|c|}
\hline & & & \\
\hline & pensar & seving & 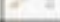 \\
\hline & conservar: & string & $2=$ \\
\hline & ecologia & binary & -7 \\
\hline & ciencis & binary & -7 \\
\hline & medioam & binary & $2=$ \\
\hline & historia. & binary & $-\quad=$ \\
\hline & bicgrafia & binary & 28 \\
\hline & arte: & binary & $2=$ \\
\hline & zcologia & binary & $x^{2}=$ \\
\hline & argaitectura & binary & $+2=$ \\
\hline & idioms & binary & $2=$ \\
\hline & discurso & string & $+2=$ \\
\hline 03 & Add fired & & \\
\hline
\end{tabular}

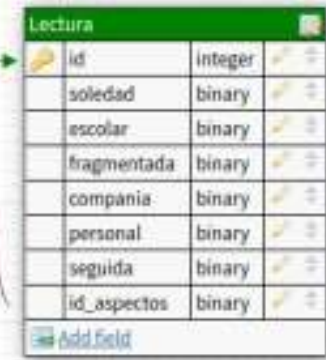

Fuente: Elaboración propia 
En este sentido, aquel que visita este repositorio podrá ver un gran número de libros de no ficción y podrá filtrar según los ítems para realizar una búsqueda más exhaustiva. Además, cuando cliquee en un libro tendrá toda la información sobre este (formato, precio, autor, características internas...) -vid. Figura 4-.

\section{Figura 4}

Interfaz del repositorio
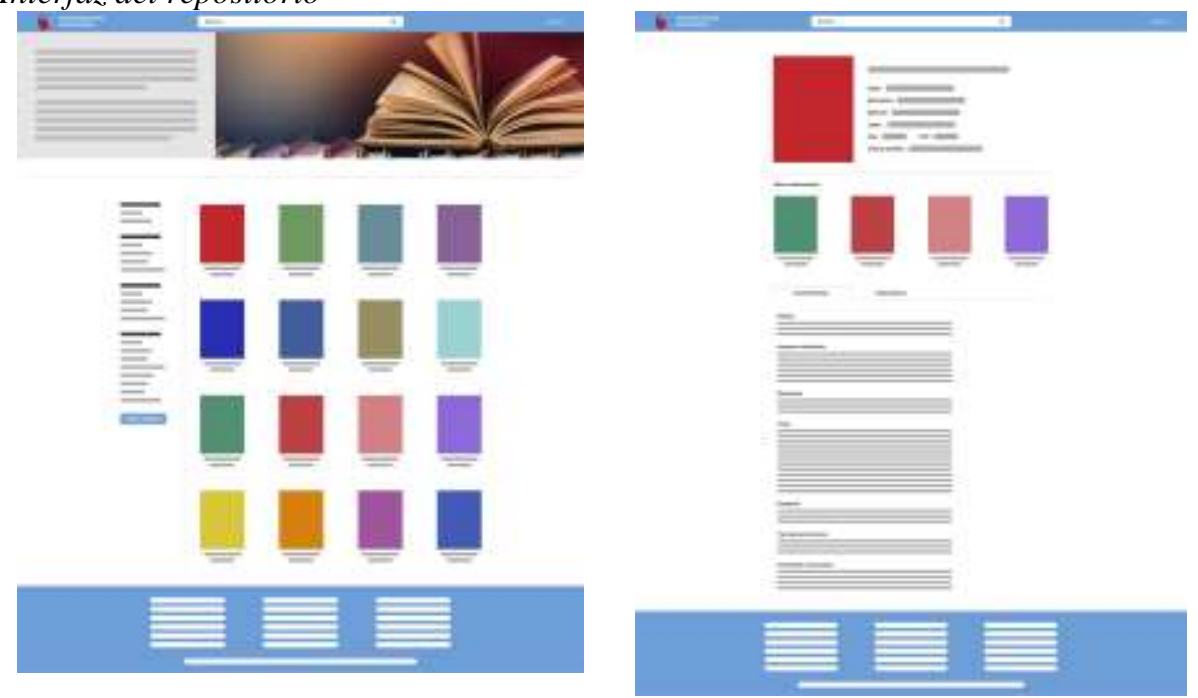

Fuente: Elaboración propia 


\section{Conclusiones}

El objetivo principal de este trabajo se centraba en el diseño y validación de una parrilla de análisis documental referida a los libros ilustrados de no ficción que sirviera de base para la creación de un repositorio digital para uso de la sociedad. Nos planteábamos aportar un instrumento que resultara útil y práctico para los agentes implicados en la formación de lectores. Pues, si bien es cierto que algunas investigaciones venían aportando estrategias para llevar estos libros al aula (Bortnem, 2008; Ruth, 2009; White, 2011; Kuhn et al., 2017; Heredia y Sánchez-Herrero, 2020, entre otros), no se contaba con un instrumento que permitiera sistematizar las características de este tipo de libros: fisicidad, estrategias de manipulación, presentación del discurso, epitextos...

Retomando la idea de Vigne (2017) acerca de la calidad de los libros editados, reflexionamos acerca de la vertiginosa proliferación de los libros ilustrados de no ficción editorial y tomamos consciencia de que para lograr un efectivo acercamiento de la lectura es necesario contar con un adecuado criterio de selección de obras $\mathrm{y}$, en consecuencia, con instrumentos que permitan analizar, de manera sistemática, los libros de no ficción.

De acuerdo con lo anterior, para establecer las dimensiones de análisis, ha sido necesaria una profunda revisión de la literatura científica relacionada con el libro-objeto, en general y con los libros ilustrados de no ficción, en particular (Garralón, 2013; Lartitegui, 2018; Tabernero, 2019). Además, se ha contado con el criterio de personas expertas en el ámbito que han aportado su visión crítica, lo que, junto al proceso de validación estadística, ha permitido configurar un instrumento fiable y extrapolable a otros contextos de investigación.

En este sentido, consideramos que la investigación en ciencias sociales debe plantearse dar respuesta a los retos que la sociedad va imponiendo. Así, con este trabajo, inserto en una investigación superior en torno al libro ilustrado de no ficción -Proyecto I+D+I RTI2018- 
093825-B-I00 Formar lectores en la sociedad digital desde el libro de no ficción-, cuyas líneas conceptuales para la formación de docentes e investigadores se recogen en Tabernero (2021), hemos cubierto un vacío en el panorama de investigación nacional en torno al libro ilustrado de no ficción pues no solo nos hemos aproximado a las características propias de estos libros, sino también hemos aportado estrategias para su análisis crítico y explotación didáctica.

Unido a esto, y con el fin de difundir nuestra investigación a mayor escala, estamos trabajando en el diseño de un repositorio digital, dinámico, que permitirá a los usuarios no solo obtener información sobre los libros más adecuados a sus intereses, sino también aportar sus conocimientos, proponiendo nuevas obras y estrategias de explotación didáctica. Este repositorio permitirá establecer un diálogo colaborativo entre la comunidad investigadora y la sociedad, lo que dotará de pleno sentido a las reflexiones aquí planteadas.

\section{Referencias bibliográficas}

Agreda, M., Hinojo, Mª A., y Sola, J. Mª (2016). Diseño y validación de un instrumento para evaluar la competencia digital de los docentes en la Educación Superior española. Revista Pixel-Bit, 49, 3956. doi: http://doi.org/10.12795/pixelbit.2016.i49.03.

Alexander, J., y Jarman, R. (2018). The pleasures of reading non-fiction. Literacy, $52(2)$, 78-85. doi: https://doi.org/10.1111/lit.12152.

Bortnem, G. (2008). Teacher use of interactive read alouds using nonfiction in early childhood classrooms. Journal of College Teaching \& Learning, 5(12), 29-44. Recuperado de http://bit.ly/3nQqHag.

Brugarolas, C.M., y Martín, M. (2002). Los libros informativos: Una propuesta de animación a la lectura. Educación y biblioteca, 14(128), 68-70. Recuperado de https://bit.ly/3rwX3ZG.

Burgos, I. (2019). Los libros de no ficción para niños o la imposibilidad del "había una vez". Idelee Revista, 288. Recuperado de http://bit.ly/3mR2WgL. 
Cabero, J., y Barroso, J. (2013). La utilización del juicio de expertos para la evaluación de TIC: el coeficiente de competencia experta. Revista Bordón, 65(2), 25-38. Recuperado de https://bit.ly/3sD1MJo.

Campos, I. O., y Marcos, A. (2021). Exploración de la relación entre la alfabetización familiar, las TIC y la competencia lectoescritora. Tejuelo, 33, 161-184. doi: https://doi.org/10.17398/1988-8430.33.161.

Castillo-Fadić, M.N., y Sologuren-Insúa, E. (2020). Léxico frecuente, riqueza léxica y estereotipos sobre la lectura de profesores en formación. Logos: Revista de Lingüística, Filosofía y Literatura, 30(1), 69-85. doi: https://doi.org/10.15443/RL3006.

Colman, P. (2007). A New Way to Look at Literature: A Visual Model for Analyzing Fiction and Nonfiction Texts. Language Arts, 84(3), 257-268. Recuperado de https://bit.ly/3aZPcwn.

Cordón-García, J. A. (2018). Combates por el libro: inconclusa dialéctica del modelo digital. Profesional de la Información, 27(3), 467481. doi: https://doi.org/10.3145/epi.2018.may.02.

Florido, B. (2020). Cartografía de libros de no ficción para el desarrollo de un plan lector de centro. Trabajo Fin de Máster. Recuperado de https://bit.ly/3u $7 \mathrm{UbmM}$

Garralón, A. (2013). Leer y saber. Los libros informativos para niños. Madrid: Tarambana Libros.

Genette, G. (2000). La obra del arte II. La relación estética. Barcelona: Lumen.

George, D., y Mallery, P. (2003). SPSS for Windows step by step: A simple guide and reference. Boston: Allyn \& Bacon.

Granado, C. (2014). Teachers as readers: a study of the reading habits of future teachers. Culture and education, 26(1), 44-70. doi: https://doi.org/10.1080/11356405.2014.908666.

Heredia, H., y Sánchez-Herrero, S. (2020). Los libros de no ficción y el book-trailer. Una propuesta didáctica para sexto de primaria. Práticas Educativas, Memórias e Oralidades-Rev. Pemo, 2(1). Recuperado de http://bit.ly/3jJkcog.

Hughes, J. (2006). Is That a Fact? The "Real" World Through Children's Non-fiction. Canadian Children's Literature/Littérature 
canadienne pour la jeunesse, 32(1), 154-169. Recuperado de http://bit.ly/3nT1BY7.

Juárez, M. (2019). Influencia de la formación inicial del profesorado en los hábitos lectores y en el concepto de educación literaria. Investigaciones Sobre Lectura, (12), 99-115. doi: https://doi.org/10.37132/isl.v0i12.287.

Kesler, T. (2012). Evoking the World of Poetic Nonfiction Picture Books. Children's Literary Education, 43, 338-354. doi: https://doi.org/10.1007/s10583-012-9173-4.

Kuhn, K., Rausch, C., Mccarty, T., Montgomery, S., y Rule, A. C. (2017). Utilizing nonfiction texts to enhance reading comprehension and vocabulary in primary grades. Early Childhood Education Journal, 45(2), 285-296. Recuperado de https://bit.ly/38RM3hh.

Lartitegui, A. G. (2018). Alfabeto del libro de conocimientos. Paradigmas de una nueva era. Zaragoza: Pantalia publicaciones.

Li, D., Beecher, C., y Byeong-Young, C. (2018). Examining the reading of informational text in 4th grade class and its relation with students' reading performance. Reading Psychology, 39(1), 1-28. doi: https://doi.org/10.1080/02702711.2017.1361493.

Lluch, G., Tabernero, R., y Calvo, V. (2015). Epitextos virtuales públicos como herramientas para la difusión del libro. El profesional de la información, 24(6), 797-804. doi: https://doi.org/10.3145/epi.2015.nov.11.

López de Ullibarri, I. y Pita Fernández, S. (1999). Medidas de concordancia. El índice de Kappa. Cadernos de atención primaria, 6(4), 223-226.

López Noguero, F. (2002). El análisis de contenido como método de investigación. XXI: Revista de Educación, 4, 167-179. Recuperado de https://bit.ly/2QQ4TQq.

Matas, A. (2018). Diseño del formato de escala Likert: el estado de la encuesta. Revista Electrónica de Investigación Educativa, 20(1), 38-47. doi: https://doi.org/10.24320/redie.2018.20.1.1347.

Moss, B. (1991). Children's Nonfiction Trade Books: A Complement to Content Area Texts. The Reading Teacher, 45(1), 2632. 
Moss, B. (2003). Exploring the Literature of Fact: Children's Nonfiction Trade Books in the Elementary Classroom. Solving Problems in the Teaching of Literacy. Guilford: Guilford Publications Inc.

Real Academia Española (2001). Diccionario de la Lengua española (22 $2^{\mathrm{a}}$ edición). Madrid: Espasa.

Romero Oliva, M. F., Florido, B., y Heredia, H. (2021a, en prensa). Epitextos editoriales en la promoción y mediación de los libros de no ficción. Espiral. Cuadernos del profesorado, 14(29).

Romero Oliva, M. F., Trigo, E., y Heredia, H. (2021b). Libros ilustrados de no ficción y formación de lectores: un análisis desde la voz de futuros docentes. Revista Ibero-Americana de Estudos em Educação, $\quad 16 \quad$ (3), 1695-1712. doi: https://doi.org/10.21723/riaee.v16iesp.3.15306.

Romero Oliva, M. F., Trigo, E., Heredia, H., y Romero Claudio, C. (2021c). Claves e instrumentos para caracterizar un libro de no ficción. En R. Tabernero (Coord.). Los libros de no ficción en la formación lectora. De la delimitación a las propuestas de centro. Barcelona: Graò.

Romero Oliva, M. F. y Trigo, E. (2019). Entre la realidad y la experiencia en la formación de nuevos lectores. Un análisis del discurso de especialistas más allá de la propia teoría. En C. Tatoj y S. Balches (Coords.), Voces y caminos en la enseñanza de español/LE: desarrollo de las identidades en el aula (pp. 119-137). Uniwersytetu Śląskiego: Wydawnictwo Uniwersytetu Śląskiego.

Ruth, S. (2009). What teachers need to know about the "new" nonfiction. The Reading Teacher, 63(4), 260-267.

Sampériz, M., Tabernero, R., Colón, M. J., y Manrique, N. (2020). El libro de no ficción para prelectores. Análisis de las claves de construcción del discurso. Cuadernos del centro de estudios de diseño y comunicación, (124), 73-90. doi: https://doi.org/10.18682/cdc.vi124.4418.

Santos-Díaz, I. C. (2017). Desarrollo curricular y recursos educativos en las bibliotecas escolares. Investigaciones Sobre Lectura, (7), 36-54. doi: https://doi.org/10.37132/isl.v0i7.196. 
Tabernero, R. (2018). Leer el álbum desde la materialidad. La manipulación como estrategia discursiva. En R. Tabernero (Ed.), Arte y oficio de leer obras infantiles (pp. 75-86). Barcelona: Octaedro.

Tabernero, R. (2019) (Ed.). El objeto libro en el universo infantil. La materialidad en la construcción del discurso. Zaragoza: Prensas de la Universidad de Zaragoza.

Tabernero, R. (2021, en prensa) (Coord.). Los libros de no ficción en la formación lectora. De la delimitación a las propuestas de centro. Barcelona: Graò.

Teaching, T. (2019). Sites of Synergy: Strategies for Readers Navigating Nonfiction Picture Books. The Reading Teacher, 72(4), 519-522. doi: https://doi.org/10.1002/trtr.1754.

Topping, K.J. (2015). Fiction and Non-Fiction Reading and Comprehension in Preferred Books. Reading Psychology, 36(4), 350387. doi: https://doi.org/10.1080/02702711.2013.865692.

Vigne, É. (2017). El formato libro y el libro objeto. Trama \& Texturas, (32), 15-20.

White, L. (2011). The place of non-fiction texts in today's primary school. Synergy, 9(1). s/p. Recuperado de https://bit.ly/3d8cUt6

Young, T.A., Moss, B., y Cornwell, L. (2007). The classroom library: A place for nonfiction, nonfiction in its place. Reading Horizons, 48(3), 1-18. Recuperado de https://bit.ly/3fryauX. 